\title{
Genomic alterations and molecular subtypes of gastric cancers in Asians
}

\author{
Xiang S. Ye $\mathrm{e}^{1,2^{*}}$, Chunping Yu ${ }^{1}$, Amit Aggarwal ${ }^{2}$ and Christoph Reinhard ${ }^{2}$
}

\begin{abstract}
Gastric cancer (GC) is a highly heterogenic disease, and it is the second leading cause of cancer death in the world. Common chemotherapies are not very effective for GC, which often presents as an advanced or metastatic disease at diagnosis. Treatment options are limited, and the prognosis for advanced GCs is poor. The landscape of genomic alterations in GCs has recently been characterized by several international cancer genome programs, including studies that focused exclusively on GCs in Asians. These studies identified major recurrent driver mutations and provided new insights into the mutational heterogeneity and genetic profiles of GCs. An analysis of gene expression data by the Asian Cancer Research Group (ACRG) further uncovered four distinct molecular subtypes with well-defined clinical features and their intersections with actionable genetic alterations to which targeted therapeutic agents are either already available or under clinical development. In this article, we review the ACRG GC project. We also discuss the implications of the genetic and molecular findings from various GC genomic studies with respect to developing more precise diagnoses and treatment approaches for GCs.
\end{abstract}

Keywords: Gastric cancer, Cancer genome, Molecular subtyping, Heterogeneity, Oncogenic drivers, Targeted therapy

\section{Background}

Gastric cancer (GC) is the fourth most common cancer and the second leading cause of cancer death in the world [1,2]. Although GC incidence has decreased in the developed world in the last several decades, the incidence in developing countries, particularly in Asian countries, continues to rise. Annually, nearly one million new cases are diagnosed, and 72,000 people die of GC. Gastric cancer in China alone accounts for over $40 \%$ of all new cases in the world, and the mortality in China is several times higher than the global average [3-5]. The GC incidence is anticipated to continuously increase over the next 40 years in China as the population ages.

Gastric cancers show high heterogeneity and different pathobiology across geographic regions, ethnicities, and genders, which likely reflect the different etiologies leading to GC development $[6,7]$. GC is traditionally classified into two main histological subtypes, diffuse and

\footnotetext{
*Correspondence: ye_xiang_s@lilly.com

1 Lilly (China) R\&D Center, Building 8, No 338, Jia Li Lue Road, Zhanghai

Hi-Tech Park, Shanghai 201203, P.R. China

Full list of author information is available at the end of the article
}

intestinal, based on anatomic locations at the proximal and distal stomach regions, respectively. Intestinal GCs are often associated with Helicobacter pylori infection, unhealthy diet, and tobacco smoking, which are common in developing Asian countries [7]. On the other hand, diffuse GCs tend to be associated with genetic abnormalities [6]. Most GC cases in developed countries are diagnosed as the diffuse subtype, whereas most cases in Asian countries belong to the intestinal subtype [6]. The heterogeneity of GCs is further reflected by the lack of universally accepted treatment options in the world. Different countries typically adopt different treatment regimens, and several classification schemes for GCs are proposed [6, 7].

Over the last several decades, efforts to improve the clinical outcomes for GC patients, such as early detection through the national screening program and radical surgery, have improved the prognosis of GC in Japan and Korea, where a high GC incidence warrants a nation-wide screening program [6]. Currently, complete surgical resection represents the only potential curative treatment for early-stage GCs. However, the majority of GC patients present with advanced diseases at 
diagnosis in China and other developing countries that lack an advanced healthcare infrastructure, particularly in rural areas. Despite efforts in treatment standardization, chemotherapy and radiotherapy have not significantly improved the 5-year survival rate for patients with advanced (stages III and IV) diseases [1, 8]. This lack of significant progress in chemotherapy and radiotherapy is not entirely surprising as these therapies are typically indiscriminate in their activity against proliferating cells; also, these therapies were developed with no consideration of cancer heterogeneity. Individual GCs demonstrated high heterogeneity, at both the histological and molecular levels, in genomic studies. This heterogeneity undoubtedly plays an important role not only in disease progression but also in the response to therapy and subsequent emergence of resistance. For instance, in a subpopulation of GC patients with Erb-B2 receptor tyrosine kinase 2 (ERBB2) amplification, the addition of trastuzumab to standard 5-fluorouracil and platinum chemotherapy significantly improved the overall survival [9]. This finding underscores the importance of, as well as the need for, molecular characterization and subtyping of GCs to develop safer and more efficacious treatment options.

Recent next-generation sequencing and gene expression profiling studies on GC have begun to establish a comprehensive landscape of genomic alterations. They have also identified a range of actionable genetic drivers as drug targets or diagnostic biomarkers. Furthermore, the combination of global gene expression profiling with longitudinal clinical data has defined clinically relevant molecular subtypes of GC. In this article, we discuss the Asian Cancer Research Group (ACRG) GC project and highlight significant findings and molecular insights that can be used for developing more effective targeted therapies and accurate diagnostic approaches for GC.

\section{Genomic alterations in GCs in Asians}

To dissect the genomic basis and underlying genetic heterogeneity of GCs, the ACRG selected a diffuse GCenriched cohort from Samsung Medical Center, Korea. The ACRG then performed whole-genome sequencing (WGS) on 49 cases of advanced stage tumors (stage IV, 19 cases; stage III, 29 cases; and stage II, 1 case) with high tumor cell contents and matched peripheral blood samples [10]. Thirty-one tumor samples were diffuse, and 18 were intestinal, microsatellite stable (MSS) GC.

Deep WGS identified genetic alterations and further revealed mutation heterogeneity and differences between the diffuse and intestinal subtypes of GC, providing a molecular basis for their differing pathobiology and prognosis. The number of somatic variants in the individual cancer genome varies greatly, ranging from
172 to 38,328 with a median of 9036 variants per tumor. A total of 4528 somatic mutations in 2553 genes were detected, with 384 genes mutated in at least two tumors. An analysis of significantly mutated genes confirmed known mutations in GCs that had been previously identified, such as tumor protein 53 (TP53), AT-rich interactive domain 1A (ARID1A), transforming growth factor beta receptor 2 (TGFßR2), and cadherin 1 (CDH1). The analysis also uncovered new significantly mutated genes, including spectrin repeat-containing, nuclear envelope 1 (SYNE1; $n=10,20 \%)$ and transmembrane protease, serine 2 (TMPRSS2; $n=3,6 \%$ ) in this Korean cohort [10]. The biological significance of the SYNE1 and TMPRSS2 mutations in GCs remains to be explored experimentally and clinically. Recurrent SYNE1 mutations were first identified in glioblastoma multiforme (GBM) tumors [11]. SYNE1 polymorphism is associated with invasive epithelial ovarian cancer risk [12]. Interestingly, subsequent association analysis of somatic mutations and gene expression changes in GBM identified $S Y N E 1$ as a major node; SYNE1 mutations have drastic effects on the expression of 543 genes, including mismatch repair genes MutS homolog 6 (MSH6) and MutL homolog 1 (MLH1). Their effects are only second to the effects of isocitrate dehydrogenase 1 (IDH1) mutations [13]. Clonal analysis of the somatic variations revealed that the intestinal subtype of GC has significantly higher ploidy and clonality than the diffuse subtype [10]. The low clonality observed in the diffuse subtype of GC indicates the presence of considerable intra-tumor heterogeneity, which has clinical implications for the emergence of drug resistance. The results also provide a plausible explanation for the poor prognosis of the diffuse subtype of GC. The intra-tumor heterogeneity and clonality in GCs were also shown in a genomic profiling study of GC patients in North China that included WGS data of two GC patients, each with three primary tumors and two matching metastatic lymph nodes [14].

The intestinal subtype of $\mathrm{GC}$ also shows more structural variations than the diffuse subtype [10]. These changes include gene fusion, translocation, and copy number variations (CNVs). Acyl-CoA-binding domain-containing 5-zinc finger E-box-binding homeobox 1 (ACBD5-ZEB1), WD repeat-containing protein 52 (WDR52)-TGFBR2, Sine oculis-binding protein homolog-mesenchymal-epithelial transition factor (SOBP-MET), and lactation-elevated protein 1 (LACE1)-MET fusions were confirmed by reverse transcription-polymerase chain reaction (RT-PCR) and Sanger sequencing. The overexpression of epidermal growth factor receptor (EGFR), MET and ERBB2 amplifications were validated by both fluorescent in situ hybridization and immunohistochemistry. These genetic changes were largely observed in the TP53-mutant background, 
and they are mutually exclusive. In general, cancer cells of the diffuse subtype have fewer genetic alterations and are mostly diploid. Mutations in $C D H 1$, catenin alpha-1 (CTNNA1), or phosphatidylinositol-4,5-bisphosphate 3-kinase, catalytic subunit alpha (PIK3CA) are also known to be associated with the diffuse subtype of hereditary GC [10]. The high mutation load and resulting neo-antigens are implicated in the tumor immune response [15]. In agreement, recurrent programmed death-ligand $(P D-L) 1$ and $P D-L 2$ gene amplifications were identified, particularly in Epstein-Barr virus-positive $\left(\mathrm{EBV}^{+}\right)$intestinal GCs [16]. This possibility is very interesting from the medical perspective, and it has significant implications for developing effective immunotherapy for GCs. However, a recent retrospective analysis of over 1000 GC gene expression profiling data showed that GCs in Western and Asian countries differ in their immune response signatures. GCs in Western countries, which are more often of the diffuse subtype, are associated with an enrichment of tumor-infiltrating T cells [17]. Although the results of this large retrospective analysis are intriguing, they must be validated through prospective studies that are specifically designed to compare geographic effects. The ongoing development of immune checkpoint inhibitors, programmed death 1 (PD-1) and PD-L1 monoclonal antibodies, in GCs will help define how genomic alterations influence the tumor immune response.

The pathway-based analysis shows that recurrent mutations occur in various cell adhesion, axon guidance, and transforming growth factor $\beta$ (TGF $\beta$ ) pathways [10]. TGF $\beta$ signaling regulates cell proliferation and suppresses the immune response, and mutations in this pathway are also identified in other GC genomic studies $[14,18]$. It is interesting that TGF $\beta R 2$ mutations identified by the ACRG are not inactivating mutations [10]. The significance of recurrent TGF $\beta R 2$ genetic alterations in the context of immunotherapy needs to be evaluated further. Axon guidance molecules are implicated in regulating cell migration and apoptosis in cancer [19]. Mutations of these molecules were previously observed in pancreatic ductal adenocarcinoma [20]. In this Korean GC cohort, axon guidance pathway mutations in ephrins, netrins, semaphorins, and slits were highly prevalent and observed in $59 \%(n=29)$ of the analyzed tumors [10]. Mutually exclusive mutations in the ephrins and slit glycoprotein/roundabout receptor (SLIT/ROBO) pathway genes indicate that they are driver mutations in cancer development. Inactivation of slit homolog 2 (SLIT2) by RNA interference (RNAi) promotes GC cell growth that is mediated through activation of protein kinase $\mathrm{B} /$ catenin, beta 1 (AKT/CTNNB1) signaling [21]. The axon guidance signaling pathway is a potential novel cancer therapeutic target.

\section{Other genomic studies of GC}

As summarized in Table 1, a growing list of genomic studies, including the ACRG project, have been carried out independently [10,14, 16-18, 22-31]. The majority of these studies have focused on GCs in Korea [10, 17, $22-25]$, China [14, 17, 18, 26], Singapore [17, 22, 27], and Japan [28], reflecting the medical significance of GCs in these countries. The molecular findings from these studies confirm the genetic heterogeneity of GCs. They also identify a common set of genetic alterations, such as $C D H 1$ and Ras homolog family member A (RhoA) mutations, that are associated with the diffuse subtype of GCs from different geographic regions, indicating that the cancers share a common genetic origin. As $C D H 1$ and RhoA regulate cell motility, mutations in these genes offer a mechanistic basis for the highly malignant phenotype of poorly differentiated cells, including prominent infiltration and stromal induction in diffuse GCs. These genomic findings are consistent with observations that the diffuse subtype of GC is associated with genetic abnormalities, and some of these are hereditary. On the other hand, the intestinal subtype of GC is more associated with environmental factors.

However, genomic studies on GCs from China, Korea, Japan, and Russia also identified non-overlapping, significantly mutated genes or altered pathways in different cohorts. For example, the ACRG study identified SYNE1 mutations in $20 \%$ of GCs as a significantly mutated cancer gene [10], which has interesting biological implications as discussed above. A study of another Korean cohort discovered B cell lymphoma 2 like 1 (BCL2L1) amplification in $18.4 \%$ and deletion in liver cancer 1 (DLC1) mutations in $10.9 \%$ of GC cases [25]. The genomic alterations in $B C L 2 L 1$ and $D L C 1$ influence drug sensitivity in GCs. BCL2L1 amplification confers sensitivity to the BLC2L1 inhibitor when used in combination with chemotherapeutic agents. DLC1 mutations promote activation of Rho/Rho-associated protein kinase (ROCK) kinase activity and make cells sensitive to ROCK kinase inhibitors [25]. In addition, a study performed on a cohort from North China identified frequent mutations in the neuregulin 1 (NRG1) and $E R B B 4$ genes [14]. Novel genomic alterations identified by different studies further demonstrate the complexity and heterogeneity of GCs, which also prompts the speculation that additional genomic alterations remain to be identified. It should be noted that the sample sizes in these GC genomic studies are small; the samples typically include fewer than 100 cases. Only the Cancer Genome Atlas (TCGA) project profiled a larger cohort of patients $(n=295)$; $75 \%$ of the patients were Caucasians (Russians, Americans, Poles, Ukrainians, and Germans), and the remaining patients were Asians (South 


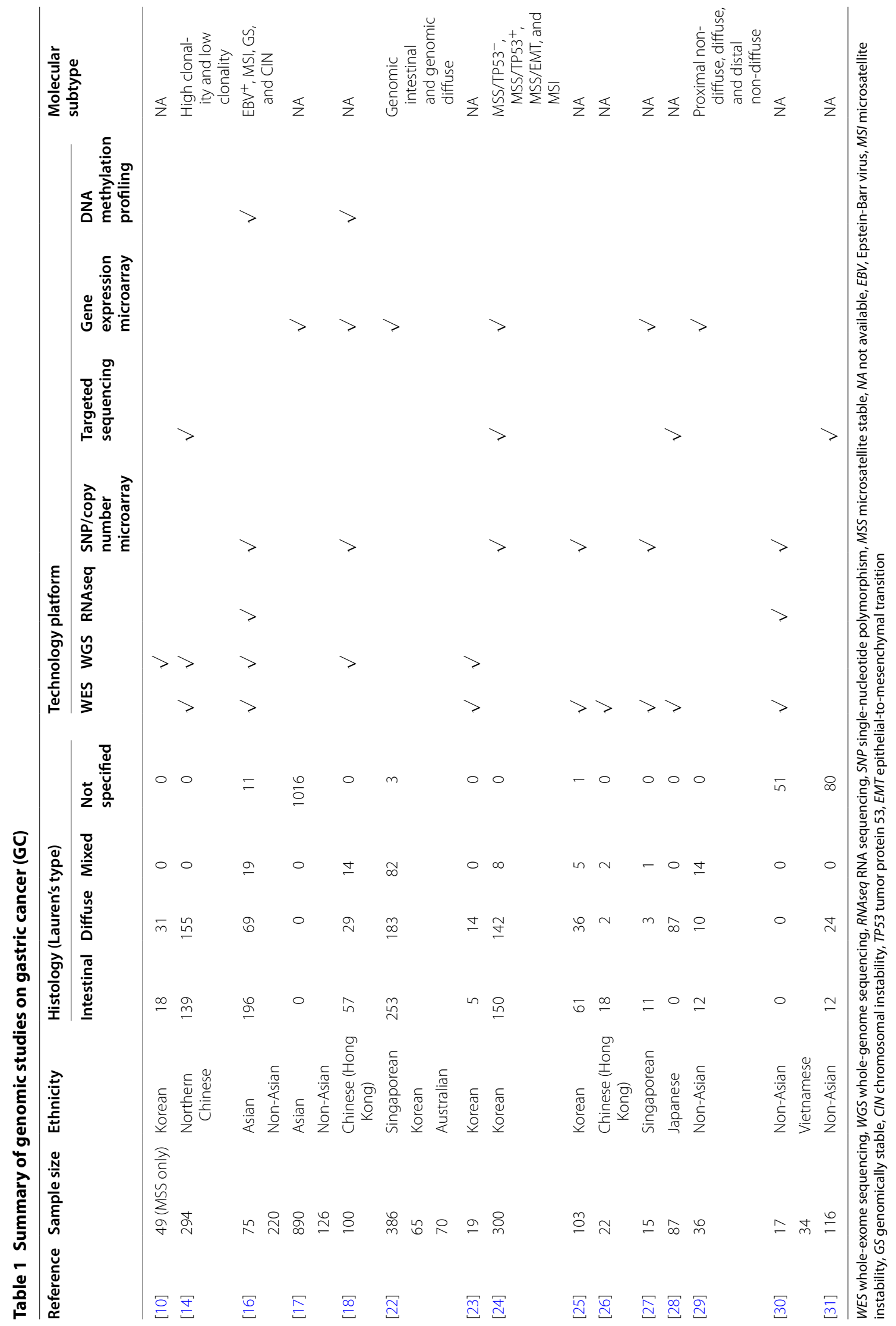


Koreans and Vietnamese) [16]. In addition to difficulty with small sample sizes, differences in the genomic technology platforms and bioinformatics pipelines may have contributed to the differences in the genomic alterations identified among the studies. Indeed, Li et al. [32] performed a retrospective, integrated analysis of 544 GC genomic data samples from previous genomic studies using an improved bioinformatics pipeline to identify significantly mutated genes. This analysis identified six previously unreported, significantly mutated genes and 12 recurrent mutated genes that exhibit higher prevalence than previously realized. These findings suggest that more profiling studies that evaluate large sample sizes for each patient population using the same advanced genomic profiling platform and bioinformatics pipeline may be needed to comprehensively determine and characterize genomic alterations in different GC cohorts.

\section{Molecular subtyping of GCs in Asians}

The high molecular heterogeneity of GCs, as demonstrated in genomic studies, further underscores the need for molecular subtyping of GCs to improve the prognosis, diagnosis, and treatment outcomes. Molecular subtyping of heterogeneous tumors, such as breast and lung cancers, has shown tremendous clinical benefits and has redefined treatment practices. Breast cancer was initially classified into four major subtypes, luminal, human epidermal growth factor 2 (HER2)-enriched, basal-like, and normal breast-like, based on the gene expression patterns according to cDNA microarrays [33]. The subtypes were subsequently further refined, and they are currently known as luminal A, luminal B, HER2-enriched, and basal-like subtypes [34]. In recent years, five novel gene expression prognostic tests for breast cancer were developed. These tests provide more reliable and reproducible results than immunohistochemistry-based assays with respect to treatment option selection [34]. The significantly mutated genes and pathways in these subtypes have further guided the development of therapies that are targeted against these genetic alterations.

In addition to the aforementioned WGS study of 49 gastric tumor specimens that assessed the mutational landscape of GC, the ACRG characterized 251 additional primary gastric tumors by gene expression profiling, genome-wide copy number microarrays, and targeted gene sequencing to identify and define clinically relevant molecular subtypes through integrated analysis of genomic alterations, survival outcome, and recurrence data [24]. The ACRG obtained 300 primary GC tumor specimens from Samsung Medical Center, which were selected on the basis of over $60 \%$ histological purity and availability of long-term followup data. Principle component (PC1-3) analysis was performed on the expression data, and the results were compared with a small pre-defined set of gene expression signatures for the epithelial-to-mesenchymal transition (EMT), microsatellite instability (MSI), cytokine signaling, cell proliferation, DNA methylation, TP53 activity, and the normal gastric tissue [35]. The analysis classified the 300 gastric tumor specimens into the following four molecular subtypes: MSI $(n=68)$, MSS/ EMT $(n=46), \mathrm{MSS} \mathrm{TP}^{2} 3^{+}(n=79)$, and MSS/TP53 ${ }^{-}$ $(n=107)$. The TCGA also classified GCs into four subtypes, including $\mathrm{EBV}^{+}$, MSI, genomically stable (GS), and chromosomal instability (CIN). Importantly, the ACRG subtype classification was also reproduced in the gene expression datasets of the TCGA cohort [16] and a Singapore cohort [36], although the proportion of each molecular subtype varied across the datasets. This variation may reflect the known geographic heterogeneity of GCs.

The ACRG molecular subtypes are associated with distinct clinical features of GC [24]. The majority of MSS/ EMT subtype GC cases $(>80 \%)$ were diagnosed as the diffuse type at stage III/IV and occurred at significantly younger ages than the other subtypes. On the other hand, the MSI subtype occurred predominantly at the antrum (75\%), and over $60 \%$ were diagnosed as the intestinal subtype and at an early stage (I/II). In addition, EBV infection occurred more frequently in the MSS/TP53 ${ }^{-}$group than in other groups. Survival analysis showed a substantial difference among four molecular subtypes; the MSS/ EMT subtype showed the worst prognosis, and the MSS subtype showed the best prognosis, which was followed by the MSS/TP53 ${ }^{+}$and MSS/TP53 ${ }^{-}$subtypes. Furthermore, the MSS/EMT group also showed a higher rate of recurrence than the MSI group. The MSS/EMT group had a higher percentage (64\%) of the first site of recurrence with peritoneal seeding and a lower percentage of liver metastasis (4.6\%) than other groups. A comparison of the ACRG subtypes [24] with the TCGA subtypes [16] showed similarities in the tumors with MSI and an enrichment of the TCGA GS, $\mathrm{EBV}^{+}$, and CIN subtypes in the ACRG MSS/EMT, MSS/TP53 ${ }^{+}$, and MSS/TP53 ${ }^{-}$ subtypes when applied to both datasets. However, the TCGA CIN and GS subtypes were present in all ACRG subtypes in the ACRG dataset. The TCGA cohort had a lower percentage of diffuse subtype cases than the ACRG cohort (24\% in TCGA vs. $45 \%$ in ACRG). Interestingly, the majority of the TCGA diffuse subtype cases (57\%) were present in the TCGA GS subtype, but only $27 \%$ of the cases were present in the ACRG MSS/EMT subtype, suggesting that the TCGA diffuse subtype cases were less heterogeneous. The analysis suggests that the ACRG 
molecular classification of GCs is unique and clinically relevant.

Targeted sequencing and CNV analysis revealed that molecular subtypes are associated with prevailing somatic alterations, many of which are clinically relevant and actionable [24]. The MSI subtype exhibited hypermutation with prevalent mutations in the Kirsten rat sarcoma viral oncogene homolog (KRAS; 23.3\%), phosphoinositide 3 kinase-phosphatase and tensin homologmechanistic target of rapamycin (PI3K-PTEN-mTOR) pathway genes $(50.6 \%)$, anaplastic lymphoma kinase (ALK; 16.3\%), and ARID1A (44.2\%). By contrast, the MSS/EMT subtype exhibited a lower number of mutations. The MSS/TP53 ${ }^{-}$subtype had more CNVs and was associated with recurrent focal amplification in ERBB2, EGFR, Cyclin E1 (CCNE1), Cyclin D1 (CCND1), murine double minute 2 ( $M D M 2$ ), roundabout homolog 2 (ROBO2), GATA-binding protein 6 (GATA6), and $\mathrm{v}$-Myc avian myelocytomatosis viral oncogene homolog (MYC). Importantly, ERBB2, EGFR, CCNE1, and CCND1 amplifications are mutually exclusive, indicating that they are driver alterations. The identification of genomic alterations and molecular subtypes, as performed in the genomic studies discussed in this article, provides important biological insight into the biology of GCs. The insight then helps guide the development of targeted therapies that can effectively treat subtypes of GC (Table 2).

\section{Conclusions}

The ACRG GC genomics project comprehensively characterized 300 patient tumors with longitudinal followup clinical data by a combination of WGS, whole-exome sequencing, $\mathrm{CNV}$ analysis, targeted sequencing, and gene expression profiling approaches [10, 24]. Together, the study further demonstrated the heterogeneity of GCs at the molecular and genetic levels, established a comprehensive mutational landscape, and defined clinically relevant molecular subtypes of GC with actionable oncogenic drivers. In fact, we found that approximately $80 \%$ of GCs in this Korean cohort harbor at least one clinically relevant genomic change [10]. Most frequent, clinically relevant alterations affect cell cycle/growth ( $p 53$, cyclindependent kinase inhibitor 2A [CDKN2A], CCND1, $C C N E 1$, aurora kinase A [AURKA], cyclin-dependent kinase 6 [CDK6], $c-M y c$, and $T G F \beta R 2)$; receptor tyrosine kinase (RTK) signaling (KRAS, neuroblastoma RAS viral oncogene homolog [NRAS], $M E T$, fibroblast growth factor receptor [FGFR], EGFR, ERBB2, PTEN, PIK3CA, and $B R a f$ ); DNA repair (breast cancer $[B R C A] 1 / 2$, ataxia telangiectasia mutated $[A T M]$, and $M D M 2)$; and epigenetics (ARID1A, mixed-lineage leukemia protein 2 [MLL2], and DNA-methyltransferase 2A [DNMT2A]). The identification of recurrent oncogenic drivers in cancer has enabled the development of a new generation of targeted cancer therapies, such as EGFR tyrosine kinase inhibitors, which have dramatically changed the treatment practices for a number of cancers [34, 37]. Many targeted cancer therapies that target these pathways are either available for other indications or under advanced clinical development. The genomic findings from these studies will inform and accelerate the development of effective targeted therapies for GCs. These genomic studies are also expected to promote comprehensive preclinical studies on molecular mechanisms underlying the pathobiology of GC in relevant disease models, such as patient-derived xenograft (PDX) models [38]. These preclinical and clinical studies will further advance our understanding of $\mathrm{GC}$ and accelerate the development of more precise diagnostic approaches and safer, more effective treatment options. Ultimately, personalized medicine will be used to improve the outcomes of individual GC patients. We are currently in a new, exciting era of GC research, including drug development, because of the rapid advancement in genomic technologies.

Table 2 Summary of clinically relevant and actionable genomic alterations and molecular subtypes of GC

\begin{tabular}{|c|c|c|c|}
\hline Subgroup & Key mutations & Key CNV events & Drugs in development \\
\hline MSI & $\begin{array}{l}\text { KRAS, PIK3CA (H1047R), PTEN, } \\
\text { mTOR, ARID1A }\end{array}$ & Very few alterations & $\begin{array}{l}\text { MEK-ERK and PI3K-mTOR pathway inhibitors, } \\
\text { immunotherapies }\end{array}$ \\
\hline MSS/EMT & PIK3CA, RhoA & CCNE1 & PI3K-mTOR, CDK2, and ROCK inhibitors \\
\hline MSS/TP53- & TP53 & $\begin{array}{l}\text { CCND1, CCNE1, ERBB2, } \\
\text { EGFR, KRAS, MYC }\end{array}$ & $\begin{array}{l}\text { RTK-focused agents, MEK-ERK, CDK4/6, } \\
\text { and CDK2 inhibitors }\end{array}$ \\
\hline MSS/TP53+ & ARID1A, PIK3CA (E542/545K) & CCNE1, KRAS & $\begin{array}{l}\text { MEK-ERK, CDK2, and PI3K-mTOR pathway } \\
\text { inhibitors }\end{array}$ \\
\hline
\end{tabular}

CNV copy number variation, KRAS kirsten rat sarcoma viral oncogene homolog, PIK3CA phosphatidylinositol-4,5-bisphosphate 3-kinase, catalytic subunit alpha, PTEN phosphatase and tensin homolog, mTOR mechanistic target of rapamycin, ARID1A AT-rich interactive domain $1 \mathrm{~A}, M E K$ mitogen-activated protein kinase kinase, ERK extracellular signal-regulated kinase, PI3K phosphoinositide 3 kinase, RhoA Ras homolog family member A, CCNE1 cyclin E1, CDK cyclin-dependent kinase, ROCK Rhoassociated protein kinase, CCND1 cyclin D1, ERBB2 Erb-B2 receptor tyrosine kinase 2, EGFR epidermal growth factor receptor, MYC v-Myc avian myelocytomatosis viral oncogene homolog, RTK receptor tyrosine kinase. Other abbreviations as in Table 1 


\section{Authors' contribution}

XSY contributed to the conceptual framework and led the preparation of the manuscript; $\mathrm{CY}$ contributed to the conceptual framework of the manuscript and prepared Table 1 and reference list; AA contributed to the conceptual framework of the manuscript and prepared Table 2; CR contributed to the conceptual framework of the manuscript; all authors participated in the preparation and revision of the manuscript. All authors read and approved the final manuscript.

\section{Author details}

1 Lilly (China) R\&D Center, Building 8, No 338, Jia Li Lue Road, Zhanghai Hi-Tech Park, Shanghai 201203, P.R. China. ${ }^{2}$ Lilly Research Laboratories, Lilly Corporate Center, Eli Lilly and Company, Indianapolis, IN 46258, USA.

\section{Acknowledgements}

We would like to thank Dr. Laura Benjamin and Dr. Sheng-Bin Peng for carefully reading the manuscript and for their helpful feedbacks.

\section{Competing interests}

The authors declare that they have no competing interests.

Received: 25 November 2015 Accepted: 4 March 2016 Published online: 09 May 2016

\section{References}

1. Takahashi T, Saikawa Y, Kitagawa Y. Gastric cancer: current status of diagnosis and treatment. Cancers. 2013;5(1):48-63.

2. Wadhwa R, Song S, Lee JS, Yao Y, Wei Q, Ajani JA. Gastric cancer-molecular and clinical dimensions. Nat Rev Clin Oncol. 2013;10(11):643-55.

3. Chen WQ, Zheng RS, Zhang SW, Li N, Zhao P, Li GL, et al. Report of incidence and mortality in China cancer registries, 2008. Chin J Cancer Res. 2012;24(3):171-80.

4. Lin Y, Ueda J, Kikuchi S, Totsuka Y, Wei WQ, Qiao YL, et al. Comparative epidemiology of gastric cancer between Japan and China. World J Gastroenterol. 2011;17(39):4421-8.

5. Chen $\mathrm{W}$, Zheng $\mathrm{R}$, Zeng $\mathrm{H}$, Zhang $\mathrm{S}$. The updated incidences and mortalities of major cancers in China, 2011. Chin J Cancer. 2015:34(11):502-7.

6. Hu B, El Hajj N, Sittler S, Lammert N, Barnes R, Meloni-Ehrig A. Gastric cancer: classification, histology and application of molecular pathology. J Gastrointest Oncol. 2012;3(3):251-61.

7. Bickenbach K, Strong VE. Comparisons of gastric cancer treatments: east vs west. J Gastric Cancer. 2012;12(2):55-62.

8. Wong HY, Yau T. Management of gastric cancer: the Chinese perspective. Transl Gastrointest Cancer. 2012;1(2):181-5.

9. Bang YJ, Van Cutsem E, Feyereislova A, Chung HC, Shen L, Sawaki A, et al. Trastuzumab in combination with chemotherapy versus chemotherapy alone for treatment of HER2-positive advanced gastric or gastrooesophageal junction cancer (ToGA): a phase 3, open-label, randomised controlled trial. Lancet. 2010;376(9742):687-97

10. Wong SS, Kim KM, Ting JC, Yu K, Fu J, Liu S, et al. Genomic landscape and genetic heterogeneity in gastric adenocarcinoma revealed by wholegenome sequencing. Nat Commun. 2014:5:5477.

11. Cancer Genome Atlas Research N. Comprehensive genomic characterization defines human glioblastoma genes and core pathways. Nature. 2008;455(7216):1061-8.

12. Doherty JA, Rossing MA, Cushing-Haugen KL, Chen C, Van Den Berg DJ, Wu AH, et al. ESR1/SYNE1 polymorphism and invasive epithelial ovarian cancer risk: an ovarian cancer association consortium study. Cancer Epidemiol Biomarkers Prev. 2010;19(1):245-50.

13. Masica DL, Karchin R. Correlation of somatic mutation and expression identifies genes important in human glioblastoma progression and survival. Cancer Res. 2011;71(13):4550-61.

14. Chen K, Yang D, Li X, Sun B, Song F, Cao W, et al. Mutational landscape of gastric adenocarcinoma in Chinese: implications for prognosis and therapy. Proc Natl Acad Sci USA. 2015;112(4):1107-12.

15. Van Allen EM, Miao D, Schilling B, Shukla SA, Blank C, Zimmer L, et al. Genomic correlates of response to CTLA-4 blockade in metastatic melanoma. Science. 2015;350(6257):207-11.
16. Cancer Genome Atlas Research N. Comprehensive molecular characterization of gastric adenocarcinoma. Nature. 2014;513(7517):202-9.

17. Lin SJ, Gagnon-Bartsch JA, Tan IB, Earle S, Ruff L, Pettinger K, et al. Signatures of tumour immunity distinguish Asian and non-Asian gastric adenocarcinomas. Gut. 2015:64(11):1721-31.

18. Wang K, Yuen ST, Xu J, Lee SP, Yan HH, Shi ST, et al. Whole-genome sequencing and comprehensive molecular profiling identify new driver mutations in gastric cancer. Nat Genet. 2014;46(6):573-82.

19. Mehlen P, Delloye-Bourgeois C, Chedotal A. Novel roles for slits and netrins: axon guidance cues as anticancer targets? Nat Rev Cancer. 2011;11(3):188-97.

20. Biankin AV, Waddell N, Kassahn KS, Gingras MC, Muthuswamy LB, Johns $A L$, et al. Pancreatic cancer genomes reveal aberrations in axon guidance pathway genes. Nature. 2012;491(7424):399-405.

21. Shi R, Yang Z, Liu W, Liu B, Xu Z, Zhang Z. Knockdown of Slit2 promotes growth and motility in gastric cancer cells via activation of AKT/betacatenin. Oncol Rep. 2014;31(2):812-8.

22. Tan IB, Ivanova T, Lim KH, Ong CW, Deng N, Lee J, et al. Intrinsic subtypes of gastric cancer, based on gene expression pattern, predict survival and respond differently to chemotherapy. Gastroenterology. 2011;141(2):476-85.

23. Lee YS, Cho YS, Lee GK, Lee S, Kim YW, Jho S, et al. Genomic profile analysis of diffuse-type gastric cancers. Genome Biol. 2014;15(4):R55.

24. Cristescu R, Lee J, Nebozhyn M, Kim KM, Ting JC, Wong SS, et al. Molecular analysis of gastric cancer identifies subtypes associated with distinct clinical outcomes. Nat Med. 2015;21(5):449-56.

25. Park H, Cho SY, Kim H, Na D, Han JY, Chae J, et al. Genomic alterations in $\mathrm{BCL} 2 \mathrm{~L} 1$ and DLC1 contribute to drug sensitivity in gastric cancer. Proc Natl Acad Sci USA. 2015;112(40):12492-7.

26. Wang K, Kan J, Yuen ST, Shi ST, Chu KM, Law S, et al. Exome sequencing identifies frequent mutation of ARID1A in molecular subtypes of gastric cancer. Nat Genet. 2011:43(12):1219-23.

27. Zang ZJ, Cutcutache I, Poon SL, Zhang SL, McPherson JR, Tao J, et al. Exome sequencing of gastric adenocarcinoma identifies recurrent somatic mutations in cell adhesion and chromatin remodeling genes. Nat Genet. 2012:44(5):570-4.

28. Kakiuchi M, Nishizawa T, Ueda H, Gotoh K, Tanaka A, Hayashi A, et al. Recurrent gain-of-function mutations of RHOA in diffuse-type gastric carcinoma. Nat Genet. 2014:46(6):583-7.

29. Shah MA, Khanin R, Tang L, Janjigian YY, Klimstra DS, Gerdes H, et al. Molecular classification of gastric cancer: a new paradigm. Clin Cancer Res. 2011;17(9):2693-701.

30. Liu J, McCleland M, Stawiski EW, Gnad F, Mayba O, Haverty PM, et al. Integrated exome and transcriptome sequencing reveals ZAK isoform usage in gastric cancer. Nat Commun. 2014:5:3830.

31. Ali SM, Sanford EM, Klempner SJ, Rubinson DA, Wang K, Palma NA, et al. Prospective comprehensive genomic profiling of advanced gastric carcinoma cases reveals frequent clinically relevant genomic alterations and new routes for targeted therapies. Oncologist. 2015;20(5):499-507.

32. Li X, Wu WK, Xing R, Wong SH, Liu Y, Fang X, et al. Distinct subtypes of gastric cancer defined by molecular characterization include novel mutational signatures with prognostic capability. Cancer Res. 2016;76(7):1724-32

33. Perou CM, Sorlie T, Eisen MB, van de Rijn M, Jeffrey SS, Rees CA, et al. Molecular portraits of human breast tumours. Nature. 2000:406(6797):747-52

34. Toss A, Cristofanilli M. Molecular characterization and targeted therapeutic approaches in breast cancer. Breast Cancer Res. 2015:17:60.

35. Benita Y, Cao Z, Giallourakis C, Li C, Gardet A, Xavier RJ. Gene enrichment profiles reveal T-cell development, differentiation, and lineage-specific transcription factors including ZBTB25 as a novel NF-AT repressor. Blood. 2010;115(26):5376-84.

36. Ooi CH, Ivanova T, Wu J, Lee M, Tan IB, Tao J, et al. Oncogenic pathway combinations predict clinical prognosis in gastric cancer. PLoS Genet. 2009:5(10):e1000676.

37. Shi Y, Au JS, Thongprasert S, Srinivasan S, Tsai CM, Khoa MT, et al. A prospective, molecular epidemiology study of EGFR mutations in Asian patients with advanced non-small-cell lung cancer of adenocarcinoma histology (PIONEER). J Thorac Oncol. 2014;9(2):154-62.

38. Zhu Y, Tian T, Li Z, Tang Z, Wang L, Wu J, et al. Establishment and characterization of patient-derived tumor xenograft using gastroscopic biopsies in gastric cancer. Sci Rep. 2015;5:8542 